Journal of Patient-Centered

$7-27-2020$

\title{
Characterizing Recurrence Following Hybrid Ablation in Patients With Persistent Atrial Fibrillation
}

David C. Kress

Lynn Erickson

Tadele W. Mengesha

David Krum

Jasbir Sra

Follow this and additional works at: https://aah.org/jpcrr

Part of the Cardiology Commons, Cardiovascular Diseases Commons, Cardiovascular System Commons, Circulatory and Respiratory Physiology Commons, Surgery Commons, and the Surgical Procedures, Operative Commons

\section{Recommended Citation}

Kress DC, Erickson L, Mengesha TW, Krum D, Sra J. Characterizing recurrence following hybrid ablation in patients with persistent atrial fibrillation. J Patient Cent Res Rev. 2020;7:227-38. doi: 10.17294/

2330-0698.1744

Published quarterly by Midwest-based health system Advocate Aurora Health and indexed in PubMed Central, the Journal of Patient-Centered Research and Reviews (JPCRR) is an open access, peer-reviewed medical journal focused on disseminating scholarly works devoted to improving patient-centered care practices, health outcomes, and the patient experience. 


\title{
Characterizing Recurrence Following Hybrid Ablation in Patients With Persistent Atrial Fibrillation
}

\author{
David C. Kress, MD, ${ }^{1}$ Lynn Erickson, MS, ${ }^{2}$ Tadele W. Mengesha, MS, ${ }^{2}$ David Krum, MS, ${ }^{2}$ \\ Jasbir Sra, MD ${ }^{1}$ \\ ${ }^{1}$ Aurora Cardiovascular and Thoracic Services, Aurora Sinai/Aurora St. Luke's Medical Centers, Milwaukee, WI; \\ ${ }^{2}$ Advocate Aurora Research Institute, Advocate Aurora Health, Milwaukee, WI
}
Purpose It is widely accepted that atrial fibrillation (AF) accounts for half of arrhythmia recurrences following endocardial catheter ablation of AF. An epicardial-endocardial approach (hybrid) has emerged as an alternative to endocardial ablation alone for the treatment of AF, yet recurrence after a hybrid procedure has not been well characterized. This retrospective study is aimed at characterizing recurrence following hybrid ablation for patients with persistent AF.

Methods Patients with persistent AF $(\mathrm{N}=108)$ received both endocardial and epicardial ablation of the posterior left atrial wall using catheter ablation and a small midline surgical approach (hybrid). Presence of atrial flutter or AF was determined with ambulatory monitoring $(n=22)$ or electrocardiogram analysis $(n=86)$ at each follow-up visit. Recurrence mode was confirmed by electrophysiology study for those patients undergoing subsequent catheter ablation after hybrid ablation.

Results Patients were followed for a mean \pm standard deviation of $25 \pm 14$ months. Of patients who had a recurrence, $53 \%(n=33)$ were in atrial flutter and $47 \%(n=29)$ were in AF. Of those who had a recurrence with atrial flutter, 14 received repeat ablation for either left $(n=11)$ or left/right $(n=3)$ atrial flutter and 3 received AF ablation. Half of ablations for atrial flutter recurrence following the hybrid procedure involved the mitral isthmus.

Conclusions Atrial flutter accounts for about half of arrhythmia recurrences post-hybrid ablation. If catheter ablation of the mitral isthmus is considered during the hybrid procedure to prevent subsequent occurrence of perimitral flutter, bidirectional block must be performed to ensure a complete line of block. ( $\mathrm{J}$ Patient Cent Res Rev. 2020;7:227-238.)

Keywords atrial fibrillation; atrial flutter; catheter ablation; hybrid; surgical ablation; mitral isthmus; recurrence

I $\mathrm{t}$ is estimated that there are 33 million people worldwide living with atrial fibrillation (AF). ${ }^{1}$ AF has been shown to increase the risk of stroke, ${ }^{2}$ sudden death, ${ }^{3}$ and heart failure, ${ }^{4}$ and patients with AF suffer from a lower quality of life. ${ }^{5}$ Antiarrhythmic drug therapy is an option for treating patients with paroxysmal AF, but for patients in persistent or longstanding persistent $\mathrm{AF}$, catheter ablation is effective $\mathrm{e}^{6-8}$

Correspondence: Jasbir Sra, MD,

Aurora St. Luke's Medical Center, 2801 W. Kinnickinnic

River Parkway, \#880, Milwaukee, WI 53215

(publishing1@aurora.org) and may reverse left atrial (LA) electrophysiological remodeling and improve left ventricular ejection fraction (LVEF). ${ }^{9}$

Studies have shown that pulmonary vein (PV) isolation (PVI) is effective in treating paroxysmal AF by targeting the AF triggers in the PV region. ${ }^{10,11}$ Yet, it has been shown that patients in persistent and long-standing persistent AF respond more favorably to an approach that includes PVI plus further substrate modification of the posterior LA wall. ${ }^{12}$

The surgical Cox-maze procedure was developed in the late $1980 \mathrm{~s}$ as a technique to interrupt electrical 
impulses and restore sinus rhythm, ${ }^{13,14}$ and evolution of this approach has led to the most recent version of the procedure, Cox-maze IV, which uses bipolar radiofrequency energy to simplify and shorten the procedure. ${ }^{15}$ While these procedures are highly effective in treating $\mathrm{AF}$, they typically require pleural access and lack the option of using electrophysiology study to uncover the presence of atrial tachycardias (AT) that may exist in the patient.

Alternatively, Wolf et $\mathrm{a}^{16}$ and Edgerton et $\mathrm{a}^{17}$ described effective and minimally invasive modified surgical ablation techniques using pleural access epicardial isolation of the PVs; however, the FAST trial demonstrated that, although the procedure is highly effective, surgical ablation had a significantly higher procedural adverse event rate than did catheter ablation. ${ }^{18}$

A minimally invasive convergent, or hybrid, approach has been developed that utilizes both epicardial ablation of the posterior surface of the LA as well as transvenous endocardial catheter ablation and has been demonstrated to be effective in the treatment of AF. ${ }^{19}$ The hybrid approach was shown to be successful in the challenging setting of persistent or long-standing persistent $\mathrm{AF}$, and a study by our center demonstrated that patients receiving the hybrid procedure had fewer repeat ablations and less recurrence at 16 months. ${ }^{20}$ It has been shown that atrial flutter (AFL) encompasses approximately half of recurrences when simple endocardial ablation techniques are used;21-23 furthermore, treating recurring $\mathrm{AF}$ in this population can be relatively challenging. ${ }^{24}$ Thus, our center sought to characterize arrhythmia recurrence after hybrid ablation in the setting of persistent and long-standing persistent AF.

\section{METHODS \\ Study Population}

The local institutional review board reviewed this protocol and determined it to be exempt status. Data from 108 consecutive patients undergoing the hybrid procedure with and without prior endocardial catheter ablation from June 2010 through March 2016 were retrospectively collected. All patients had persistent or long-standing persistent AF, as defined by the 2017 expert consensus statement on AF ablation, ${ }^{21}$ for a median of 12 (interquartile range: 6-34) months. Mean age was $61 \pm 10$ years, with a mean follow-up of $25 \pm$ 14 months. Baseline characteristics are summarized in Table 1.

Table 1. Baseline Characteristics

\begin{tabular}{|c|c|c|c|c|}
\hline Characteristic & $\begin{array}{l}\text { Overall } \\
(N=108)\end{array}$ & $\begin{array}{c}\text { With prior } \\
\text { ablation }(n=60)\end{array}$ & $\begin{array}{l}\text { Without prior } \\
\text { ablation }(n=48)\end{array}$ & $\boldsymbol{P}$ \\
\hline Age & $61.1 \pm 9.7$ years & $60.7 \pm 8.5$ years & $61.6 \pm 10.9$ years & 0.448 \\
\hline Sex & & & & 0.654 \\
\hline Female & $14(13.0 \%)$ & $7(11.7 \%)$ & $7(16.6 \%)$ & \\
\hline Male & $94(87.0 \%)$ & $53(88.3 \%)$ & $41(85.4 \%)$ & \\
\hline Body mass index & $34.6 \pm 6.2 \mathrm{~kg} / \mathrm{m}^{2}$ & $33.2 \pm 5.3 \mathrm{~kg} / \mathrm{m}^{2}$ & $36.2 \pm 6.9 \mathrm{~kg} / \mathrm{m}^{2}$ & 0.016 \\
\hline Hypertension & $70(64.8 \%)$ & $36(60.0 \%)$ & $34(70.8 \%)$ & 0.241 \\
\hline Coronary artery disease & $22(20.4 \%)$ & $9(15.0 \%)$ & $13(27.1 \%)$ & 0.121 \\
\hline Diabetes mellitus & $15(13.9 \%)$ & $7(11.7 \%)$ & $8(16.7 \%)$ & 0.455 \\
\hline Prior stroke, TIA, or thromboembolism & $10(9.3 \%)$ & $5(8.3 \%)$ & $5(10.4 \%)$ & 0.711 \\
\hline $\mathrm{CHA}_{2} \mathrm{DS}_{2}$-VASc score & $1.8 \pm 1.2$ & $1.5 \pm 1.1$ & $2.1 \pm 1.3$ & 0.010 \\
\hline LVEF & $54.7 \% \pm 10.8 \%$ & $55.5 \% \pm 9.6 \%$ & $53.8 \% \pm 12.2 \%$ & 0.707 \\
\hline Left atrial size & $5.2 \pm 0.7 \mathrm{~cm}$ & $5.0 \pm 0.7 \mathrm{~cm}$ & $5.3 \pm 0.7 \mathrm{~cm}$ & 0.060 \\
\hline Duration of persistent AF & $12(6-34)$ months & 12 (6-36) months & $12(6-34)$ months & 0.582 \\
\hline
\end{tabular}

Values are reported as $n(\%)$, mean \pm standard deviation, or median (interquartile range).

$A F$, atrial fibrillation; LVEF, left ventricular ejection fraction; TIA, transient ischemic attack. 


\section{Anesthesia and Monitoring}

Anesthesia and monitoring were performed as previously described. ${ }^{20}$ General anesthesia was induced in all patients in the electrophysiology operating room. Hemodynamic monitoring was performed throughout the procedure, and transesophageal echocardiography was used to exclude thrombus in the LA appendage. Esophageal temperature monitoring was used throughout the entire procedure.

\section{Hybrid Ablation}

Patients undergoing the hybrid procedure had epicardial ablation concomitant with endocardial ablation, as described hereafter. Patients underwent epicardial ablation of the posterior LA wall using a transabdominal endoscopic approach followed by endocardial catheter ablation, with the goal of posterior wall isolation. ${ }^{20}$ Briefly, a $12-\mathrm{mm}$ port through a $2-\mathrm{cm}$ midline incision and two 5-mm ports were placed for instruments and carbon dioxide insufflation. A 30- or 40-cm cannula (Subtle, AtriCure, Inc.) was placed through a $2-\mathrm{cm}$ opening in the central tendon of the diaphragm and guided into the oblique sinus with a $6.5-\mathrm{mm}$ scope. A preablation epicardial map of the posterior LA and proximal PV trunks was created for all patients after March 2014 using a 3D mapping system (CARTO $3^{\circledR}$, Biosense Webster, Inc.) under direct visualization. Each lesion was delivered with a 3-cm probe (Numeris ${ }^{\circledR}$, AtriCure), which was saline-irrigated and vacuum-attached, at 30 watts for 90 seconds. All lesions were delivered with cold saline infusion within the pericardial sac. Lesions were repeated, if necessary, to achieve a cumulative delivery of 2700 Joules. Esophageal temperature was monitored to avoid an increase of more than $0.5^{\circ} \mathrm{C}$ during any lesion. In the event of temperature rise exceeding $0.5^{\circ} \mathrm{C}$, the power was interrupted sufficiently long for the temperature to return to baseline, often with the instillation of additional cold saline into the pericardial sac. The entire posterior LA from the right PV trunk to the left PV trunk was ablated with 2 rows of parallel overlapping lesions (Figure 1). The anterior left inferior PV trunk was ablated. Lesions were placed in the recess between the inferior vena cava and right inferior PV in some patients. A new acquisition of voltage signals was completed to create a post-epicardial ablation voltage map in those patients with pre-epicardial ablation mapping. Areas that failed to show electrical silence underwent repeat ablation to achieve a silent final postablation map. A 19-mm drain (BLAKE ${ }^{\circledR}$, Ethicon, Inc.) was placed into the pericardial cavity. The abdominal incisions were closed in a standard fashion.

Immediately following epicardial ablation, endocardial ablation was performed as previously described by Kress et al. ${ }^{20}$ In brief, after obtaining venous access, a 20-pole diagnostic catheter (St. Jude Medical) was advanced under fluoroscopic guidance into the coronary sinus. Diagnostic quadripolar catheters were positioned at the right atrial appendage and His bundle positions. Upon completion of all sheath insertions, 5000 units of heparin was given. An additional 5000 units was given via the transseptal sheath after puncture. During the catheter ablation procedure, infusion of heparin was maintained to achieve an activated clotting time of 300 to 350 seconds.

Fluoroscopy and either transesophageal or intracardiac echocardiography were performed to guide the sheath (PREFACE ${ }^{\circledR}$, Medtronic) for transseptal puncture. An esophageal probe was placed to monitor temperature throughout the entire endocardial ablation procedure.

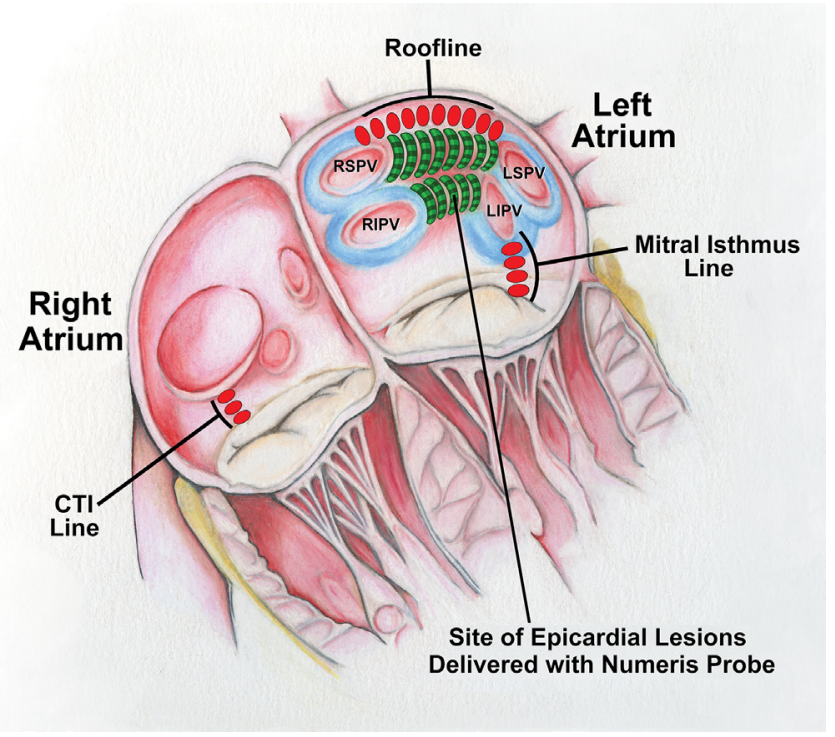

Figure 1. Cutaway view of the left and right atria of the heart. CTI, cavotricuspid isthmus; LIPV, left inferior pulmonary vein; $L S P V$, left superior pulmonary vein; RIPV, right inferior pulmonary vein; $R S P V$, right superior pulmonary vein. 
Using the CARTO 3D mapping system, maps of the left and right atria were created for identifying fractionated electrograms and any AT or AFL.

The PVs were checked for electrical activity and isolated as needed. If the PVs were silent from previous ablation, PVI was not performed. PVI, when needed, was achieved using either conventional catheter ablation or the cryoballoon (Medtronic), and isolation of the PVs was confirmed with a mapping and ablation catheter. Radiofrequency catheter ablation was used to ablate complex fractionated atrial electrograms in the LA if they were present, and linear lesions were applied at the discretion of the physician performing the catheter ablation if a reentrant tachycardia occurred during the procedure. Linear ablation involved some or all of the following: a line connecting the mitral annulus to the left superior PV, the roofline connecting the superior veins, the mitral annulus to the right superior PV, and the cavotricuspid isthmus line on the right side of the atrium. Ablation in the coronary sinus was performed in patients not initially demonstrating conduction block across the mitral isthmus line or having fractionated electrogram recordings in the coronary sinus. Sinus rhythm was the procedural endpoint in all cases. Ablation of the most common lesions is illustrated in Figure 1.

Demonstration of conduction block was attempted in all linear lesions and confirmed during pacing from the LA appendage for both mitral isthmus and rooflines $^{25}$ as well as through CARTO 3D analysis of the scar. If AT or AFL developed at any point during the initial ablation procedure, mapping and ablation of the AT/AFL was performed and conduction block confirmed across the linear lesion. For all patients, if tachycardia persisted following delivery of all lesions, patients were converted to sinus rhythm using intravenous infusion of ibutilide (1 mg over 10 minutes) and/or direct-current cardioversion. Heparin was partially reversed with protamine following the procedure.

\section{Follow-up Visits}

Patient follow-up was conducted as part of the standard of care, as previously described. ${ }^{20}$ Data related to procedure complications occurring within 30 days of ablation, as defined by 2017 expert consensus, ${ }^{21}$ were collected. Cardiac symptom data and need for antiarrhythmic drugs were collected from each patient follow-up visit. Nonambulatory and/or ambulatory data were collected from each follow-up visit or device interrogation following the hybrid procedure. Typically, patients were followed at 1, 3, 6, 9, and 12 months, or any time symptoms were noted.

Detectable AF/AFL/AT was evaluated with a minimum of a 30-second electrocardiogram recording or rhythm strip or with remote and/or in-person ambulatory monitoring, including pacemaker interrogation, loop recorder, activated clotting time monitor, and Holter monitor. Recurrence was defined as documented AF, AFL, or AT occurring after the 3-month blanking period or a repeat ablation or atrioventricular junction ablation with permanent pacemaker at any time throughout the follow-up period, regardless of antiarrhythmic drug use. Initial intervention for recurrence was treated at the physician's discretion based on symptoms, patient history, and clinical condition.

\section{Characterizing Recurrence and Repeat Ablation Following the Hybrid Procedure}

If repeat ablation was not the treatment for recurrence, diagnosis of AF, AFL, or AT was based off monitoring device records reviewed at the time of recurrence. For patients demonstrating both AF and AFL, recurrence was treated statistically as AF for the purposes of this study. For patients having an electrophysiology study/repeat ablation following the hybrid procedure, cardiac mapping, entrainment, and pacing maneuvers were used to characterize the anatomical location of the AFL during the procedure. Upon repeat ablation, the PVs were checked for electrical isolation using mapping and catheter techniques for every repeat ablation procedure. During repeat ablation, the PVs found to have electrical activity were isolated by either radiofrequency or cryoballoon.

\section{Statistical Analysis}

SAS 9.4 software (SAS Institute Inc.) was used for statistical analysis. Continuous variables are reported as mean \pm standard deviation or median (interquartile range), as appropriate. Categorical variables are presented as frequency count (percentage). Comparisons of characteristics for patients who had prior ablation versus no prior ablation were made using 
the Wilcoxon rank-sum test for continuous variables and chi-squared or Fisher's exact tests for categorical variables. Comparisons of the time to recurrence for patients with and without implantable devices were made with a Student's paired $t$-test.

\section{RESULTS}

\section{Procedural Results}

Procedural results are outlined in Table 2. In summary, the median total hybrid procedure time for both epicardial and endocardial ablation was 308 (278-352) minutes. Overall, 85.2\% of patients required endocardial PVI during the hybrid procedure, and those with prior ablation required significantly less endocardial PVI than those without prior ablation $(75.0 \%$ vs $97.9 \% ; \mathrm{P}<0.001)$. Patients with ablation prior to the hybrid procedure also required cardioversion to restore sinus rhythm during the procedure significantly less often than did those with no prior ablation $(26.7 \%$ vs $45.8 \%$; $\mathrm{P}=0.038)$. Endocardial linear lesions, including the mitral isthmus line $(67.6 \%)$, roofline $(52.8 \%)$, mitral annulus to right superior PV (19.4\%), and cavotricuspid isthmus line $(38.0 \%)$, were performed in $95(88 \%)$ patients.

\section{Time to Recurrence}

Patients were monitored for recurrence following the hybrid procedure using either ambulatory $(\mathrm{n}=22)$ or nonambulatory $(n=86)$ methods. Of the ambulatory methods used, 18 patients had continuous monitoring for more than 48 hours with either permanent pacemaker $(n=9)$, implantable loop recorder $(n=6)$, or implantable cardioverter-defibrillator $(n=3)$.

In the overall population, mean days to recurrence was $396 \pm 324$. Patients who received continuous ambulatory monitoring for their arrhythmia following the hybrid ablation were also studied separately to help aid the analysis. Of the 18 patients who had continuous monitoring with permanent pacemaker, implantable loop recorder, or implantable cardioverter-defibrillator, 14 had recurrence as defined by more than 30 seconds of arrhythmia at some point in their follow-up.

Patients with continuous implantable devices $(\mathrm{n}=18)$ recurred, on average, at $265 \pm 226$ days, and those without continuous monitoring from an implantable device $(n=90)$ recurred at a mean of $434 \pm 340$ days.

Table 2. Procedural Characteristics

\begin{tabular}{|c|c|c|c|c|}
\hline Procedural data & $\begin{array}{l}\text { Overall } \\
(\mathrm{N}=108)\end{array}$ & $\begin{array}{c}\text { With prior } \\
\text { ablation }(n=60)\end{array}$ & $\begin{array}{c}\text { Without prior } \\
\text { ablation }(n=48)\end{array}$ & $P$ \\
\hline Endocardial PVI required & $92(85.2 \%)$ & $45(75.0 \%)$ & $47(97.9 \%)$ & $<0.001$ \\
\hline Cryoballoon ablation used & $78(72.2 \%)$ & $36(60.0 \%)$ & $42(87.5 \%)$ & 0.002 \\
\hline Linear lesions & $95(88.0 \%)$ & $53(88.3 \%)$ & $42(87.5 \%)$ & 0.894 \\
\hline Roofline & $57(52.8 \%)$ & $32(53.3 \%)$ & $25(52.1 \%)$ & 0.897 \\
\hline Mitral isthmus & $73(67.6 \%)$ & $40(66.7 \%)$ & $33(68.8 \%)$ & 0.818 \\
\hline Cavotricuspid isthmus line & $41(38.0 \%)$ & $22(36.7 \%)$ & $19(39.6 \%)$ & 0.756 \\
\hline Mitral annulus to RSPV & $21(19.4 \%)$ & $11(18.3 \%)$ & $10(20.8 \%)$ & 0.744 \\
\hline DCCV required & $38(35.2 \%)$ & $16(26.7 \%)$ & $22(45.8 \%)$ & 0.038 \\
\hline Ibutilide required & $33(30.6 \%)$ & $16(26.7 \%)$ & $17(35.4 \%)$ & 0.327 \\
\hline Procedural complications & $12(11.1 \%)$ & $7(11.7 \%)$ & $5(10.4 \%)$ & 0.837 \\
\hline Radiation dose, mGy & 573.5 (289-932) & $469.0(260-886)$ & $597.0(322-1129)$ & 0.190 \\
\hline \multicolumn{5}{|l|}{ Time, minutes } \\
\hline Cryoballoon ablation & $26.5(0-32)$ & $23.5(0-32)$ & $27.5(21-32)$ & 0.164 \\
\hline Radiofrequency ablation & $24.6(16-43.2)$ & $28.6(14.5-50.8)$ & $24.2(16.7-38.3)$ & 0.208 \\
\hline Total procedure & $308.0(278-352)$ & $308.0(268-346)$ & $308.0(278.5-355.5)$ & 0.694 \\
\hline Fluoroscopy & $54.0(43.2-73.8)$ & $53.0(40.2-76.6)$ & $55.6(44.2-68.1)$ & 0.861 \\
\hline
\end{tabular}

Values are reported as $n$ (\%) or median (interquartile range).

$D C C V$, direct-current cardioversion; PVI, pulmonary vein isolation; RSPV, right superior pulmonary vein. 
There was no significant difference between the time to recurrence in patients with and without an implantable device $(\mathrm{P}=0.9)$.

\section{Rate of 12-Month Recurrence and Effect of Prior Ablation on Outcome}

Overall, $64.2 \%$ of patients had arrhythmia-free survival at 12 months, as defined by no AF, AFL, or AT. Of all the patients who underwent the hybrid procedure, $57.4 \%$ were on an antiarrhythmic drug at 12 months. There was no significant difference in arrhythmia-free survival among patients with and without ablation prior to the hybrid procedure (Figure 2 ). The 12-month arrhythmia-free survival estimate following hybrid ablation and up to 1 additional ablation for AFL was $70.0 \%$ (Figure 3). There also was no significant difference in estimated AF-free survival among patients with and without prior ablation (83.0\% vs $82.0 \%$ ). Overall, AF-free survival was estimated to be $82.6 \%$ at 12 months (Figure 4 ).

\section{Mode of Recurrence and Initial Intervention Following Hybrid Ablation}

Following hybrid ablation and spanning the entirety of the follow-up period, $53.2 \%(n=33)$ of patients who recurred at any time were in AFL and 46.8\% $(n=29)$ were in AF. Of these 62 total patients who had recurrence over some period during their follow-up, $66.1 \%(n=41)$ were on an antiarrhythmic drug at the time of recurrence. Looking at the subpopulation of those who were monitored with implantable devices, of the patients who recurred at any time $(\mathrm{n}=14), 8$ (57.1\%) recurred with AF and 6 (42.9\%) with AFL. Similarly, of those without implantable devices who had recurrence $(n=48), 21(43.8 \%)$ had AF and 27 (56.3\%) had AFL.

Initial intervention for recurrence of both $\mathrm{AF}$ and $\mathrm{AFL}$ is outlined in Figure 5. The initial intervention for $\mathrm{AF}$ and AFL patients, respectively, was as follows: $37.9 \%$ $(n=11)$ and $24.2 \%(n=8)$ medication change; $20.7 \%$ $(\mathrm{n}=6)$ and $30.3 \%(\mathrm{n}=10)$ direct-current cardioversion; $6.9 \%(\mathrm{n}=2)$ and $27.3 \%(\mathrm{n}=9)$ repeat ablation; $3.4 \%$ $(\mathrm{n}=1)$ and $6.1 \%(\mathrm{n}=2)$ rate control; $10.4 \%(\mathrm{n}=3)$ and $0 \%$ atrioventricular junction ablation; and $20.7 \%(\mathrm{n}=6)$ and $12.1 \%(\mathrm{n}=4)$ no intervention.

Repeat Ablations/Electrophysiology Study for AFL: Of the 14 patients undergoing a repeat ablation for their AFL at any time following the hybrid procedure, all were found to have a left-sided flutter component (Table 3). Of the 14 confirmed AFL recurrences, 9 $(64.3 \%)$ occurred in patients with a catheter ablation prior to the hybrid procedure. All the catheter ablations prior to the hybrid procedure were for AF and had a PVI component except for one, which was performed owing to right-sided AFL. Seven of the confirmed 14 flutters involved the perimitral isthmus (as defined by Matsuo et $\mathrm{al}^{26}$ ). Of the 7 confirmed AFL recurrences that contained a perimitral component, $6(85.7 \%)$ had the mitral isthmus line as part of the hybrid ablation procedure. Other areas of AFL occurrence included the

Table 3. Mode of Recurrent AFL Ablation in Patients With and Without Ablation Prior to Hybrid Procedure Who Required Repeat Ablation Subsequent to the Hybrid Procedure

\begin{tabular}{lccc}
\hline Repeat AFL ablation location* & $\begin{array}{c}\text { Overall } \\
(\mathbf{N}=14)\end{array}$ & $\begin{array}{c}\text { With prior } \\
\text { ablation }(\mathbf{n = 9})\end{array}$ & $\begin{array}{c}\text { Without prior } \\
\text { ablation }(\mathbf{n = 5})\end{array}$ \\
\hline Perimitral, $\mathrm{n}(\%)$ & $4(29 \%)$ & $3(75 \%)$ & $1(25 \%)$ \\
Left atrial roof, $\mathrm{n}(\%)$ & $3(22 \%)$ & $3(100 \%)$ & $0(0 \%)$ \\
Perimitral and right atrium, $\mathrm{n}(\%)$ & $2(14 \%)$ & $0(0 \%)$ & $2(100 \%)$ \\
Perimitral and left atrial roof, $\mathrm{n}(\%)$ & $1(7 \%)$ & $1(100 \%)$ & $0(0 \%)$ \\
LSPV and LAA base, $\mathrm{n}(\%)$ & $1(7 \%)$ & $1(100 \%)$ & $0(0 \%)$ \\
Posterior wall, $\mathrm{n}(\%)$ & $1(7 \%)$ & $1(100 \%)$ & $0(0 \%)$ \\
Multiple left, $\mathrm{n}(\%)$ & $2(14 \%)$ & $0(0 \%)$ & $2(100 \%)$ \\
\hline
\end{tabular}

*Location of AFL was determined by electrophysiology study conducted during the repeat AFL ablation. This table represents AFL ablations that followed the hybrid ablation, regardless of timing of the follow-up intervention.

$A F L$, atrial flutter; LAA, left atrial appendage; LSPV, left superior pulmonary vein. 


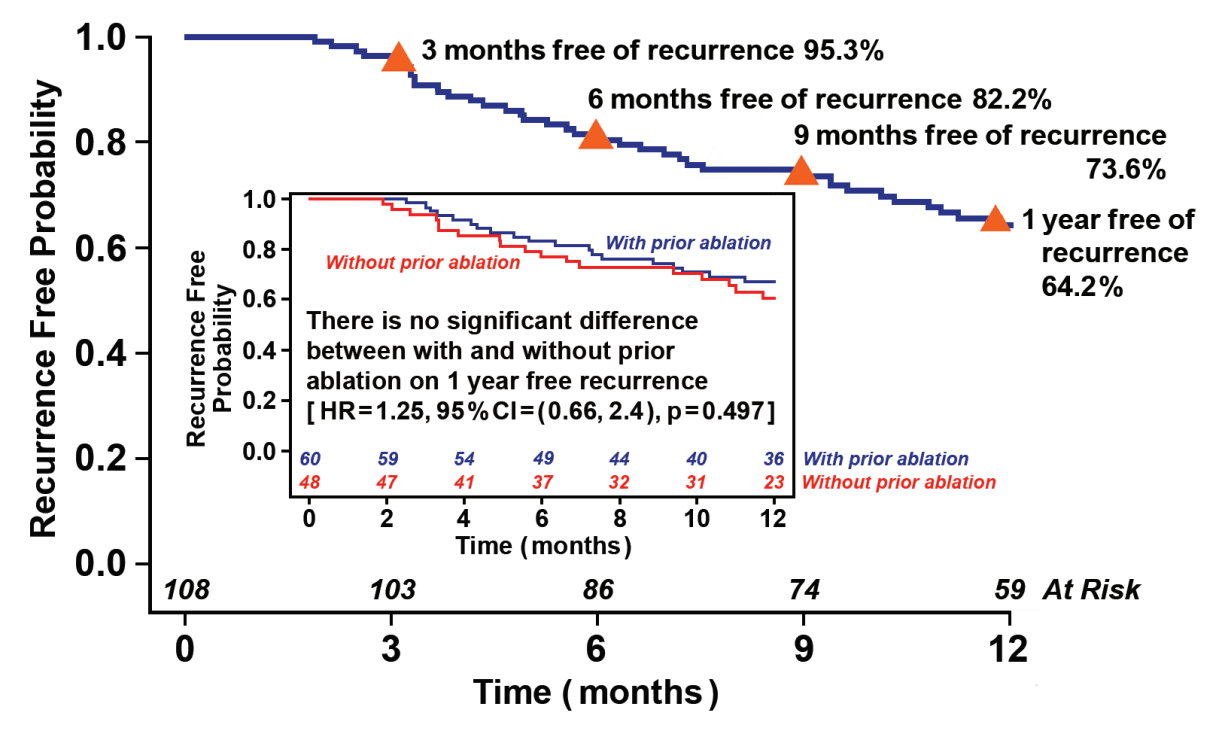

Figure 2. Kaplan-Meier curve for 12-month arrhythmia-free (atrial fibrillation/flutter/tachycardia) survival for a single hybrid ablation procedure. HR, hazard ratio.

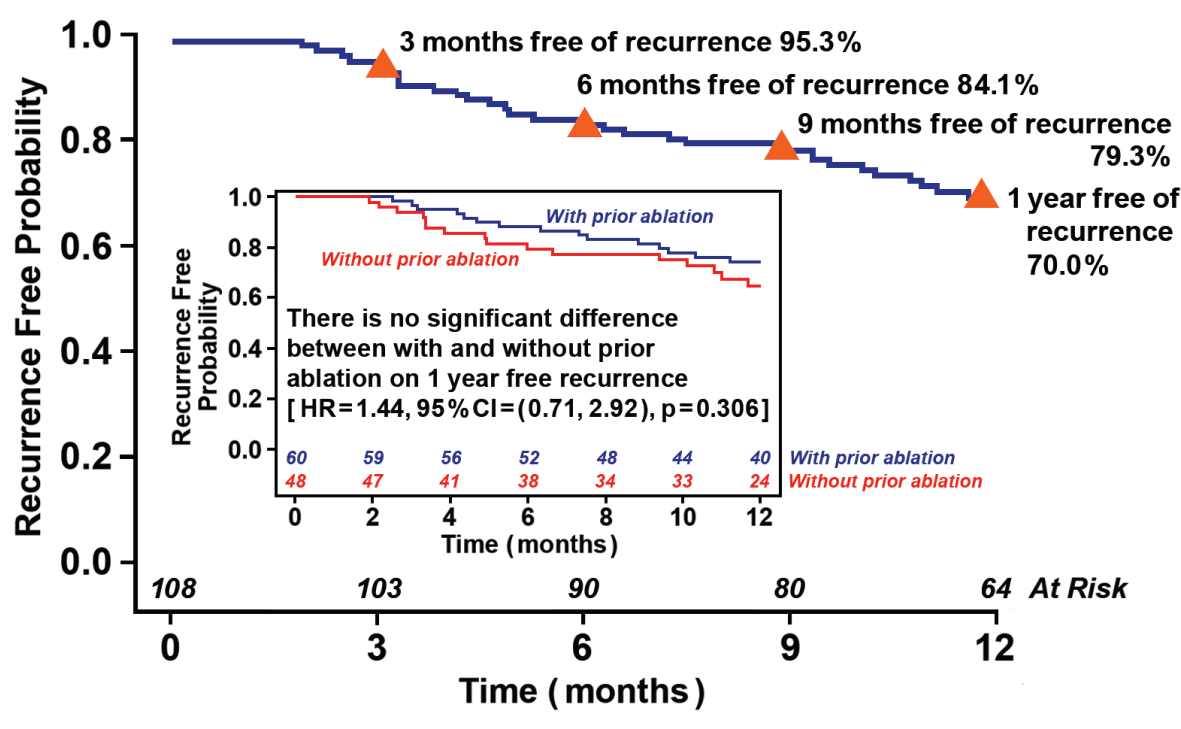

Figure 3. Kaplan-Meier curve for 12-month arrhythmia-free survival following hybrid ablation, allowing for 1 additional atrial flutter ablation. The first occurrence of flutter was not counted as an event if it was successfully ablated in a single additional procedure. HR, hazard ratio.

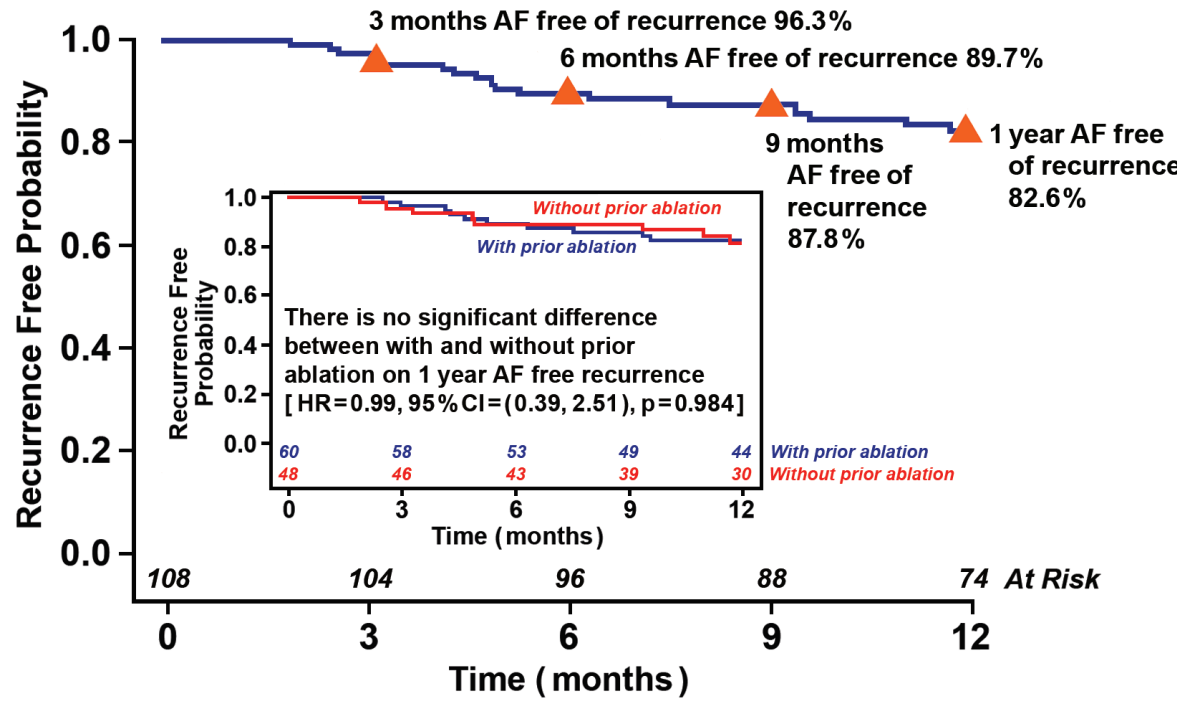

Figure 4. Kaplan-Meier curve for 12-month atrial fibrillation (AF)-free survival following a single hybrid ablation procedure. HR, hazard ratio. 


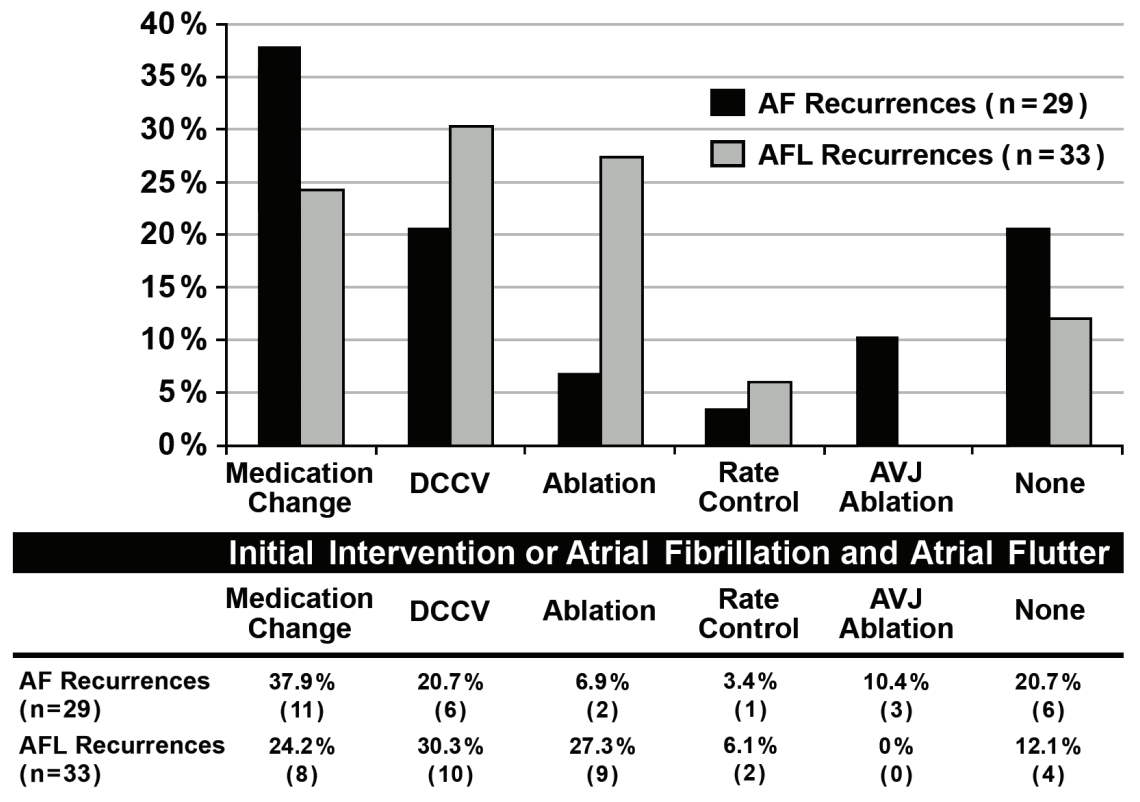

Figure 5. Initial intervention for recurrences following the hybrid ablation procedure. AF, atrial fibrillation; AFL, atrial flutter; AVJ, atrioventricular junction; DCCV, direct-current cardioversion.
LA roof ( $n=3)$, the left superior PV and base of the LA appendage $(n=1)$, and the LA posterior wall $(n=1) ; 1$ case had multiple locations in the LA.

Two patients (14.3\%) requiring repeat ablation and electrophysiology study for AFL required some additional radiofrequency ablation to re-isolate PVs that demonstrated electrical activity, and 12 patients (85.7\%) demonstrated silent PVs upon repeat ablation and electrophysiology study. The posterior wall was silent in 8 of these 14 patients (57.1\%), as demonstrated by CARTO 3D, and 6 patients $(42.9 \%)$ demonstrated areas of fractionated electrograms on their posterior wall that required catheter ablation.

Repeat Ablations/Electrophysiology Study for AF: Of the 3 patients who underwent a repeat ablation for their AF at any time following the hybrid procedure, 1 patient required radiofrequency ablation of the right inferior PV to re-isolate the vein. Other veins previously isolated in this patient showed silence according to CARTO 3D. This same patient required additional ablation in the left septal region anterior to the right superior PV and LA appendage.

The PVs of the other 2 patients who had repeat AF ablation and electrophysiology study demonstrated silence. In one case, the posterior wall demonstrated mainly scar, but the LA required catheter ablation for the roofline, lateral isthmus line, and multiple fractionated areas. This patient had no ablation prior to the hybrid procedure. The roofline and mitral isthmus line were completed as part of the hybrid procedure. The other repeat ablation also had electrically silent PVs from hybrid ablation but required ablation of the mitral isthmus line, right superior PV region, roofline, anterior mitral line from the right superior PV to the roof, and the cavotricuspid isthmus line. This patient had no ablation prior to the hybrid procedure.

\section{Complications}

There were 8 complications noted in the study cohort. Three patients suffered from groin complication as follows: 1 received a right femoral embolectomy, 1 had a right groin bleed within 48 hours of the procedure, and 1 had a pseudoaneurysm of the groin requiring thrombin injection. One patient suffered from pericarditis and a nonocclusive pulmonary embolus in 1 of the left pulmonary branches. There were 2 late tamponade/pericardial effusions, 1 cerebrovascular accident, and 1 death due to a gastrointestinal bleed 2 weeks following the procedure. The patient was on warfarin at the time of the bleed.

\section{DISCUSSION}

In this retrospective analysis, we aimed to describe the manner with which recurrence occurred following the minimally invasive convergent, or hybrid, ablation 
procedure for persistent and long-standing persistent AF. This series was performed in a high-volume center with experienced operators. The population included patients both with and without catheter ablation prior to the hybrid procedure. Although data are reported as a heterogeneous population using either radiofrequency or cryoballoon ablation to isolate the PVs as part of the endocardial procedure, in a randomized multicenter study, the cryoballoon was found noninferior to radiofrequency ablation in PVI. ${ }^{27}$ All baseline characteristics were similar except the patients who had no prior ablation had a significantly higher $\mathrm{CHA}_{2} \mathrm{DS}_{2}$-VASc score, putting them at a higher risk of stroke prior to the hybrid ablation compared with patients who did undergo prior ablation.

\section{Time to Recurrence}

Overall mean time to recurrence was slightly over 12 months for the entire population. Although not statistically significant, patients with continuous monitoring of $>48$ hours had a shorter time to recurrence compared with those with no ambulatory monitoring, most likely owing to the ability of continuous monitoring to pick up small disturbances in rhythm. Since this study defines arrhythmia of $>30$ seconds as recurrence, there is a greater likelihood of having a reportable recurrence with continuous monitoring, although this number was not statistically significant. Analysis, especially that which is retrospective in nature, is oftentimes limited by follow-up monitoring tools, and there may be concern regarding nonambulatory monitoring results. Yet, there is also a challenge when studying those with continuous monitoring, owing to the incessant nature of persistent AF. While the 2017 expert consensus statement recommends centers report recurrence as anything exceeding 30 seconds of $\mathrm{AF}$, they also encourage centers to report other endpoints, such as AF burden reduction or quality of life. This is due to the oftentimes noncurative nature of persistent $\mathrm{AF}$ and the fact that Kaplan-Meier curve analysis of this strict definition could underestimate the benefit of ablation for persistent $\mathrm{AF}^{21}$

\section{Arrhythmia-Free Survival Outcomes}

Patients with persistent or long-standing persistent $\mathrm{AF}$ may be a challenging population to treat with ablation. Wokhlu et al have described that persistent $\mathrm{AF}$ is a predictor of overall recurrence and very late recurrence. ${ }^{24}$ In a large randomized trial of 589 patients, Verma et al described $49 \%$ and $46 \%$ freedom from $\mathrm{AF}$ with PVI and complex fractionated atrial electrograms or PVI and linear lesions, respectively. ${ }^{28}$ At our center, the overall AF-free survival at 12 months was $82.6 \%$ and the 12-month arrhythmia-free survival, including both AF and AFL recurrence, following hybrid ablation was $64.2 \%$. Allowing for 1 additional ablation for AFL following the hybrid procedure, the 12-month arrhythmia-free survival was $70 \%$ in this cohort. Our results are noteworthy for persistent and long-standing persistent AF patients given the lower predictive value of nonpersistent $\mathrm{AF}$ and lower rates of ablation success in prior studies. ${ }^{29,30}$ Our analysis of 12-month outcomes also aligns with recent similar studies by de Asmundis et al and Maesen et al. ${ }^{22,23}$ This is important, as we are a large-volume center with experienced operators performing the hybrid procedure and our population reported is roughly double that of both similar papers, further validating their results. We also have the additional benefit of prior ablation Kaplan-Meier curve analysis, but we caution that the numbers may not be high enough to see a difference statistically between the two groups, as discussed henceforth.

\section{Effect of Prior Ablation on Acute Hybrid Procedure Outcome}

Those patients with prior ablation required significantly less frequent endocardial PVI during the hybrid procedure. This observation is most likely due to previously isolated PVs from the prior ablation or from the epicardial ablation portion of the hybrid procedure - most likely the isolation occurred with the former since epicardial ablation using this technique does not aim to isolate the PVs. Since preand postablation epicardial mapping did not occur until later in the data set, it is difficult to establish whether the PVs were isolated from previous ablation or with epicardial ablation itself. Patients with prior ablation also converted to sinus rhythm with ablation during the hybrid procedure significantly more frequently than those without prior ablation. This may suggest that prior ablation has a cumulative effect on outcomes, as seen with Maesen et al, ${ }^{23}$ but this requires further analysis.

Results of this study show that ablation prior to the hybrid procedure does not affect the probability 
of recurrence-free outcome at 12 months in this population. This finding should be studied further in a larger cohort to assess the importance of prior ablation on hybrid procedure outcomes.

\section{Mode of Recurrence and Initial Intervention Following Hybrid Ablation}

We found that slightly over half of all recurrences following hybrid ablation were AFL, which is similar to the statement made in the 2017 expert consensus document: "ATs of new onset make up to $50 \%$ of all arrhythmias observed following catheter-based ablation of AF." 21 From our results, it seems that recurrence following hybrid ablation does not largely involve reconnection of the PVs.

Studies have shown that AFL recurrences following endocardial ablation often involve focal AT originating from PV ostial reconnection or macroreentrant flutters occurring around anatomical scarring of the LA. ${ }^{31,32}$ In the case of macroreentrant flutters, Chae and colleagues found that $42 \%$ of recurrent ATs could be ablated at the mitral isthmus, with the LA roof and septum accounting for $20 \%$ and $13 \%$ of reentrant tachycardia, respectively. ${ }^{32}$ It is suggested that these common postablation recurrences could be reduced or prevented by demonstration of complete block or avoidance of linear lesions altogether. ${ }^{32}$ A recent similar study from de Asmundis and colleagues reported that about half of the AFL recurrences involve the mitral isthmus line. ${ }^{22}$ Similarly, at our center, AFL accounted for 53\% of recurrences, and of those, $50 \%$ were perimitral or had a perimitral component, verifying further that the mitral isthmus is a difficult area to ablate.

The subpopulation analysis of those who had a recurrence of AFL or AF with or without continuous monitoring looked like that of the overall population, suggesting that although the study lacks a large population with continuous monitoring, results may have been similar.

Overall, initial intervention for AF recurrence relied more heavily on medication changes, atrioventricular junction ablation, or no intervention than did the AFL recurrences. AFL recurrences had more directcurrent cardioversion, repeat ablation, and rate control methods as their initial intervention. This may be due to the stringent definition of recurrence that was used in the study and the patient's ability to convert spontaneously, with a dose change or with a change in antiarrhythmic medication, while in AF for a very short period. Ablation of AFL may have been used more often because of the greater ability to treat the recurrence with a repeat ablation using linear lesions. Most recurrences following the hybrid procedure did not require PVI because the PVs were deemed silent. Many patients receiving an ablation following the procedure received radiofrequency ablation for complex fractionated atrial electrograms seen in the LA on electrophysiology study and/or AFL.

Our data suggest that during the hybrid procedure, if the endocardial mitral isthmus line is created, conduction block must be confirmed to optimize its postprocedural scar transmurality and gap absence. This will reduce the incidence of developing new perimitral AFL. If there is a history of previous ablation of the mitral isthmus, the scar should be evaluated for conduction gaps, and ablation to close the gaps should be performed during the endocardial portion of the procedure. There were multiple flutters seen in 6 of the 14 patients with repeat AFL ablation $(42.9 \%)$ following the hybrid procedure, emphasizing the importance of entrainment mapping of multiple flutters upon recurrence as suggested by Chae et al. ${ }^{32}$

\section{Limitations}

The study was retrospective in nature, and ambulatory monitoring was used in a minority of the patients. The electrophysiological evaluation of postablation arrhythmias was limited to patients deemed symptomatic enough to undergo a followup catheter ablation and therefore did not include less symptomatic patients. Detection of postablation arrhythmias was done at follow-up intervals that were based on standard of care, emergency department, or acute care visits.

\section{CONCLUSIONS}

Atrial flutter accounts for about half of arrhythmia recurrences in the first year following a hybrid ablation procedure. Knowledge of the recurrence mechanism is important for physicians to consider when treating a patient with this procedure so that perimitral and other types of flutter might be avoided. 


\section{Patient-Friendly Recap}

- Hybrid ablation, or ablation of the heart's left atrium using both catheter and surgical techniques, has emerged as an option for treating persistent atrial fibrillation, a complicated cardiac condition.

- After analyzing past cases, the authors found that in patients who experience a recurrent arrhythmia following the hybrid procedure, half have what is called atrial flutter and half of those flutters stem from the mitral isthmus region of the left atrium.

- Having knowledge of the anatomical site where recurrence is likely to originate allows physicians to consider that region when performing hybrid ablation, especially in those patients who have undergone a previous ablation procedure.

\section{Acknowledgments}

The authors thank Jennifer Pfaff and Susan Nord of Aurora Cardiovascular and Thoracic Services for editorial preparation of the manuscript and Brian Schurrer and Brian Miller of Aurora Cardiovascular and Thoracic Services for assistance with the figures.

\section{Author Contributions}

Study design: Kress, Erickson, Mengesha, Sra. Data acquisition or analysis: Erickson, Mengesha. Manuscript drafting: Kress, Erickson, Krum, Sra. Critical revision: Kress, Erickson.

\section{Conflicts of Interest}

Dr. Kress served as chair of the Data and Safety Monitoring Board for the Converge trial (sponsor: nContact Inc. and, subsequently, AtriCure) during the years 2014-2018. He was a consultant with Clinipace Inc. to serve in this role, not of the sponsors.

\section{References}

1. Chugh SS, Havmoeller R, Narayanan K, et al. Worldwide epidemiology of atrial fibrillation: a Global Burden of Disease 2010 Study. Circulation. 2014;129:837-47. CrossRef

2. Kannel WB, Wolf PA, Benjamin EJ, Levy D. Prevalence, incidence, prognosis, and predisposing conditions for atrial fibrillation: population-based estimates. Am J Cardiol. 1998;82(8A):2N-9N. CrossRef

3. Chen LY, Benditt DG, Alonso A. Atrial fibrillation and its association with sudden cardiac death. Circ J. 2014;78:2588-93. CrossRef

4. Wang TJ, Larson MG, Levy D, et al. Temporal relations of atrial fibrillation and congestive heart failure and their joint influence on mortality: the Framingham Heart Study. Circulation. 2003;107:2920-5. CrossRef
5. Dorian P, Jung W, Newman D, et al. The impairment of health-related quality of life in patients with intermittent atrial fibrillation: implications for the assessment of investigational therapy. J Am Coll Cardiol. 2000;36:1303-9. CrossRef

6. Parkash R, Tang AS, Sapp JL, Wells G. Approach to the catheter ablation technique of paroxysmal and persistent atrial fibrillation: a meta-analysis of the randomized controlled trials. J Cardiovasc Electrophysiol. 2011;22:729-38. CrossRef

7. Oral H, Pappone C, Chugh A, et al. Circumferential pulmonary-vein ablation for chronic atrial fibrillation. $N$ Engl J Med. 2006;354:934-41. CrossRef

8. Mont L, Bisbal F, Hernández-Madrid A, et al. Catheter ablation vs. antiarrhythmic drug treatment of persistent atrial fibrillation: a multicentre, randomized, controlled trial (SARA study). Eur Heart J. 2014;35:501-7. CrossRef

9. Pump A, Di Biase L, Price J, et al. Efficacy of catheter ablation in nonparoxysmal atrial fibrillation patients with severe enlarged left atrium and its impact on left atrial structural remodeling. J Cardiovasc Electrophysiol. 2013;24:1224-31. CrossRef

10. Haïssaguerre M, Jaïs P, Shah DC, et al. Spontaneous initiation of atrial fibrillation by ectopic beats originating in the pulmonary veins. N Engl J Med. 1998;339:659-66. CrossRef

11. Packer DL, Kowal RC, Wheelan KR, et al. Cryoballoon ablation of pulmonary veins for paroxysmal atrial fibrillation: first results of the North American Arctic Front (STOP AF) pivotal trial. J Am Coll Cardiol. 2013;61:1713-23. CrossRef

12. Haïssaguerre M, Hocini M, Sanders P, et al. Catheter ablation of long-lasting persistent atrial fibrillation: clinical outcome and mechanisms of subsequent arrhythmias. J Cardiovasc Electrophysiol. 2005;16:1138-47. CrossRef

13. Cox JL, Canavan TE, Schuessler RB, et al. The surgical treatment of atrial fibrillation. II. Intraoperative electrophysiologic mapping and description of the electrophysiologic basis of atrial flutter and atrial fibrillation. J Thorac Cardiovasc Surg. 1991;101:406-26. CrossRef

14. Cox JL, Schuessler RB, Boineau JP. The surgical treatment of atrial fibrillation. I. Summary of the current concepts of the mechanisms of atrial flutter and atrial fibrillation. $J$ Thorac Cardiovasc Surg. 1991;101:402-5. CrossRef

15. Gaynor SL, Diodato MD, Prasad SM, et al. A prospective, single-center clinical trial of a modified Cox maze procedure with bipolar radiofrequency ablation. J Thorac Cardiovasc Surg. 2004;128:535-42. CrossRef

16. Wolf RK, Schneeberger EW, Osterday R, et al. Video-assisted bilateral pulmonary vein isolation and left atrial appendage exclusion for atrial fibrillation. J Thorac Cardiovasc Surg. 2005;130:797-802. CrossRef

17. Edgerton JR, Jackman WM, Mack MJ. A new epicardial lesion set for minimal access left atrial maze: the Dallas lesion set. Ann Thorac Surg. 2009;88:1655-7. CrossRef

18. Boersma LV, Castella M, van Boven W, et al. Atrial fibrillation catheter ablation versus surgical ablation treatment (FAST): a 2-center randomized clinical trial. Circulation. 2012;125:23-30. CrossRef

19. Pison L, La Meir M, van Opstal J, Blaauw Y, Maessen J, Crijns HJ. Hybrid thoracoscopic surgical and transvenous catheter ablation of atrial fibrillation. J Am Coll Cardiol. 2012;60:54-61. $\underline{\text { CrossRef }}$

20. Kress DC, Erickson L, Choudhuri I, et al. Comparative effectiveness of hybrid ablation versus endocardial catheter ablation alone in patients with persistent atrial fibrillation. JACC Clin Electrophysiol. 2017;3:341-9. CrossRef 
21. Calkins H, Hindricks G, Cappato R, et al. 2017 HRS/EHRA/ ECAS/APHRS/SOLAECE expert consensus statement on catheter and surgical ablation of atrial fibrillation. Heart Rhythm. 2017;14:e275-444. CrossRef

22. de Asmundis C, Varnavas V, Sieira J, et al. Two-year followup of one-stage left unilateral thoracoscopic epicardial and transcatheter endocardial ablation for persistent and long-standing persistent atrial fibrillation. J Interv Card Electrophysiol. 2019 Sep 13 [Epub ahead of print]. CrossRef

23. Maesen B, Pison L, Vroomen M, et al. Three-year follow-up of hybrid ablation for atrial fibrillation. Eur J Cardiothorac Surg. 2018;53(suppl_1):i26-32. CrossRef

24. Wokhlu A, Hodge DO, Monahan KH, et al. Long-term outcome of atrial fibrillation ablation: impact and predictors of very late recurrence. $J$ Cardiovasc Electrophysiol. 2010;21:1071-8. CrossRef

25. Jaïs $\mathrm{P}$, Hocini M, Hsu LF, et al. Technique and results of linear ablation at the mitral isthmus. Circulation. 2004;110:29963002. CrossRef

26. Matsuo S, Wright M, Knecht S, et al. Peri-mitral atrial flutter in patients with atrial fibrillation ablation. Heart Rhythm. 2010;7:2-8. CrossRef

27. Kuck KH, Brugada J, Furnkranz A, et al. Cryoballoon or radiofrequency ablation for paroxysmal atrial fibrillation. N Engl J Med. 2016;374:2235-45. CrossRef
28. Verma A, Jiang CY, Betts TR, et al. Approaches to catheter ablation for persistent atrial fibrillation. $N$ Engl $\mathrm{J} \mathrm{Med}$. 2015;372:1812-22. CrossRef

29. Hummel J, Michaud G, Hoyt R, et al. Phased RF ablation in persistent atrial fibrillation. Heart Rhythm. 2014;11:202-9. CrossRef

30. Bassiouny M, Saliba W, Hussein A, et al. Randomized study of persistent atrial fibrillation ablation: ablate in sinus rhythm versus ablate complex-fractionated atrial electrograms in atrial fibrillation. Circ Arrhythm Electrophysiol. 2016;9(2):e003596. CrossRef

31. Ouyang F, Antz M, Ernst S, et al. Recovered pulmonary vein conduction as a dominant factor for recurrent atrial tachyarrhythmias after complete circular isolation of the pulmonary veins: lessons from double Lasso technique. Circulation. 2005;111:127-35. CrossRef

32. Chae S, Oral H, Good E, et al. Atrial tachycardia after circumferential pulmonary vein ablation of atrial fibrillation: mechanistic insights, results of catheter ablation, and risk factors for recurrence. J Am Coll Cardiol. 2007;50:1781-7. $\underline{\text { CrossRef }}$

(C) 2020 Aurora Health Care, Inc.

\section{CME Accreditation and Credit Designation Statements}
Advocate Aurora Health is accredited by the Accreditation Council for Continuing Medical Education (ACCME) to provide continuing medical education for physicians. Aurora Health Care designates this journal-based CME article for a maximum of 1.00 AMA PRA Category $1 \mathrm{credit}^{\mathrm{TM}}$. Physicians should claim only the credit commensurate with the extent of their participation in the activity.
To earn CME credit for this article, visit https://cme.advocateaurorahealth.org/ content/characterizing-recurrence-following-hybrid-ablation-patients-persistent- atrial-fibrillation to access and complete the online quiz. 